\title{
A CAD Utilizing 3D Massive-Training ANNs for Detection of Flat Lesions in CT Colonography: Preliminary Results
}

\author{
Kenji Suzuki, PhD, Ivan Sheu, BS, Don C. Rockey ${ }^{a}$, MD, Abraham H. Dachman, MD \\ Department of Radiology, The University of Chicago, Chicago, IL 60637 \\ ${ }^{a}$ Department of Internal Medicine, University of Texas Southwestern Medical Center, TX 75390
}

\begin{abstract}
Our purpose was to develop a computer-aided diagnostic (CAD) scheme for detection of flat lesions (also known as superficial elevated or depressed lesions) in CT colonography (CTC), which utilized 3D massive-training artificial neural networks (MTANNs) for false-positive (FP) reduction. Our CAD scheme consisted of colon segmentation, polyp candidate detection, linear discriminant analysis, and MTANNs. To detect flat lesions, we developed a precise shape analysis in the polyp detection step to accommodate the analysis to include a flat shape. With our MTANN CAD scheme, 68\% (19/28) of flat lesions, including six lesions "missed" by radiologists in a multicenter clinical trial, were detected correctly, with 10 (249/25) FPs per patient.
\end{abstract}

Keywords: flat neoplasm, superficial elevated lesions, virtual colonoscopy, computer-aided diagnosis, missed polyps, massive training, artificial neural networks

\section{INTRODUCTION}

Colorectal cancer is the second leading cause of cancer deaths in the U.S. CT colonography (CTC) is a technique for detecting colorectal neoplasms by use of a CT scan of the colon. The diagnostic performance of CTC in detecting polyps (i.e., precursors of cancer), however, remains uncertain because of a propensity for perceptual errors. Computer-aided detection (CAD) of polyps has the potential to overcome this difficulty with CTC. A major challenge in CAD development is the detection of flat lesions (also known as flat polyps, superficial elevated or depressed lesions/neoplasms), because existing CAD schemes are designed for detecting the common bulbous polyp shape. Flat lesions in the colon are a major source of false-negative interpretations in CTC [1]; thus, detection of flat lesions by $\mathrm{CAD}$ is very important. Because flat lesions are also a source of false negatives in "reference-standard" optical colonoscopy [1], the detection of flat lesions would impact on colorectal cancer mortality. Some flat lesions are known to be histologically aggressive; therefore, the detection of such lesions is critical clinically $[1,2]$. One study showed that flat polyps contributed to $54 \%$ of superficial carcinomas [3].

Our purpose was to develop a CAD scheme for detection of flat lesions in CTC, which utilized 3D massive-training artificial neural networks (MTANNs) for false-positive (FP) reduction.

\section{METHODS}

\subsection{Initial Polyp-Detection Scheme}

Our initial polyp-detection scheme consists of 1) colon segmentation based on CT value-based analysis and colon tracing, 2) detection of polyp candidates based on precise shape analysis on the segmented colon, 3) calculation of 3D pattern features of the polyp candidates, and 4) linear discriminant analysis (LDA) for classification of the polyp candidates as polyps or non-polyps based on the pattern features.

\subsection{Detection of Flat Lesions}

To detect flat lesions, we developed a precise shape analysis in the polyp detection step to accommodate the analysis to include a flat shape. To characterize a polypoid and flat shape, we employed the precise shape (PS) index and curvedness [4]. The PS index determines to which of the following nine topologic shapes: spherical cap, dome, ridge, saddle ridge, saddle, saddle rut, rut, trough, o spherical cup, the object belongs. A common bulbous polyp can be identified as a spherical cap in the PS index space, but flat lesions cannot. Flat lesions would range from dome to ridge in the PS index space. For detecting both bulbous polyps and flat lesions, we used spherical cap, dome, and ridge in the

Medical Imaging 2009: Computer-Aided Diagnosis, edited by Nico Karssemeijer, Maryellen L. Giger Proc. of SPIE Vol. 7260, 72601A · C 2009 SPIE · CCC code: 1605-7422/09/\$18 · doi: 10.1117/12.811073 
PS index space. Because the PS index is independent of the scale of objects, we used curvedness (R), which represents the scale of objects. Curvedness is defined as $R(p) \equiv \sqrt{\frac{\kappa_{1}(p)^{2}+\kappa_{2}(p)^{2}}{2}}$. Because the scale of flat lesions would be larger than that of bulbous polyps, we broadened the curvedness range to include the flat-lesion scale. By use of the above precise shape analysis based on the shape and scale space $(P S, R)$, both bulbous polyps and flat lesions can be detected. We applied a fuzzy c-means clustering algorithm to the detected polyp candidates to group them. For determining the boundary of the polyp candidates, we applied the volume-growing technique based on the shape and scale space $(P S, R)$ to the grouped candidates. We used the same concept of the precise shape analysis in volumegrowing to cover both bulbous polyps and flat lesions. Because of the broadening of the shape and scale in the morphologic analysis, we expect to obtain a larger variety of FP detections, such as stool, with flat shape and blunted folds. We reduce such a variety of FPs by using a "mixture of expert" 3D MTANNs.

\subsection{Architecture of a 3D Massive-Training ANN (3D MTANN)}

To process 3D CTC volume data, we developed a 3D MTANN [5] (Fig. 1) by extending the structure of a 2D MTANN [6-10]. The 3D MTANN consists of a linear-output ANN model [11-13] which is capable of operating on voxel data directly. The 3D MTANN is trained with input CTC volumes and the corresponding "teaching" volumes for enhancement of polyps and suppression of non-polyps. The input to the 3D MTANN is the voxel values $I(x, y, z)$ in a subvolume VS extracted from an input volume. The output of the 3D MTANN is a continuous value, represented by

$$
O(x, y, z)=N N\left\{I(x-i, y-j, z-k) \mid(i, j, k) \in V_{S}\right\} \text {, }
$$

where $N N\{\bullet\}$ is the output of the linear-output ANN. The entire output volume is obtained by scanning of an input CTC volume with the 3D MTANN in 3D space.

\subsection{Training of a 3D MTANN}

For enhancement of polyps and suppression of non-polyps in CTC volumes, the teaching volume contains a 3D Gaussian distribution with standard deviation $\sigma_{T}$. This distribution represents the "likelihood of being a polyp" for a polyp and zero for a non-polyp:

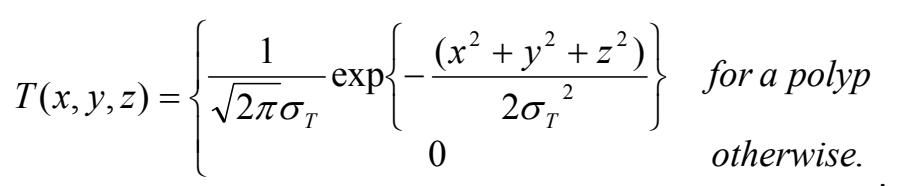

A 3D Gaussian distribution is used to approximate an average shape of polyps. The expert 3D MTANN involves training with a large number of subvolume-voxel pairs; we call it a massive-subvolumes training scheme. For enriching the training samples, a training volume, $V_{T}$, extracted from the input CTC volume is divided voxel by voxel into a large number of overlapping sub-volumes. Single voxels are extracted from the corresponding teaching volume as teaching values. The expert 3D MTANN is massively trained by use of each of a large number of the input sub-volumes together with each of the corresponding teaching single voxels; hence the term "massive-training ANN." The error to be minimized by training of the nth expert 3D MTANN is given by

$$
E_{n}=\frac{1}{P_{n}} \sum_{c} \sum_{(x, y, z) \in V_{T_{n}}}\left\{T_{n, c}(x, y, z)-O_{n, c}(x, y, z)\right\}^{2},
$$

where $c$ is a training case number, $O_{n, c}$ is the output of the nth expert MTANN for the $c$ th case, $T_{n, c}$ is the teaching value for the $n$th expert MTANN for the $c$ th case, and $P_{n}$ is the number of total training voxels in the training volume for the $n$th expert 3D MTANN, $V_{T n}$. The expert 3D MTANN is trained by a linear-output back-propagation algorithm [11]. After training, the expert 3D MTANN is expected to output the highest value when a polyp is located at the center of the sub-volume of the expert 3D MTANN, a lower value as the distance from the sub-volume center increases, and zero when the input sub-volume contains a non-polyp.

For combining output voxels from the trained expert 3D MTANNs, we developed a 3D scoring method, as shown in Fig.

2. A score for a given polyp candidate from the nth expert 3D MTANN is defined as 
$S_{n}=\sum_{(x, y, z) \in V_{E}} f_{G}\left(\sigma_{n} ; x, y, z\right) \times O_{n}(x, y, z)$

where

$f_{G}\left(\sigma_{n} ; x, y, z\right)=\frac{1}{\sqrt{2 \pi} \sigma_{n}} \exp \left\{-\frac{\left(x^{2}+y^{2}+z^{2}\right)}{2 \sigma_{n}^{2}}\right\}$

is a $3 \mathrm{D}$ Gaussian weighting function with standard deviation $\sigma_{n}$, and with its center corresponding to the center of the volume for evaluation, $V_{E}$, and $O_{n}(x, y, z)$ is the output volume of the nth trained expert 3D MTANN, where its center corresponds to the center of $V_{E}$. The use of the 3D Gaussian weighting function allows us to combine the responses (outputs) of a trained expert 3D MTANN as a 3D distribution. A 3D Gaussian function is used for scoring, because the output of a trained expert 3D MTANN is expected to be similar to the 3D Gaussian distribution used in the teaching volume. This score represents the weighted sum of the estimates for the likelihood that the volume (polyp candidate) contains a polyp near the center, i.e., a higher score would indicate a polyp, and a lower score would indicate a nonpolyp.

\subsection{Mixture of Expert MTANN Architecture}

To distinguish between polyps and various sources of FPs, we developed a "mixture of expert" 3D MTANNs [14] (Fig. 3). The mixture of expert MTANNs consisted of seven 3D MTANNs. Each of the seven MTANNs was trained independently with a different type of FPs and common typical polyps. The 3D MTANNs were combined with a mixing ANN such that all major sources of FPs such as stool with bubbles, colonic walls, bulbous-shape folds, stool with flat shape and blunted folds, and solid stool could be removed.

\subsection{Database of CTC}

Our independent database consists of 50 CTC scans obtained from a multicenter clinical trial in which 15 leading medical institutions participated nationwide [15]. Each patient was scanned in both supine and prone positions with a multi-detector-row CT system with collimations of 1.0-2.5 mm and reconstruction intervals of 1.0-2.5 mm. Each CT slice has a spatial resolution of $0.5-0.7 \mathrm{~mm} /$ pixel. A radiologist experienced in CTC $(>1000$ cases read $)$ determined the locations of polyps with reference to colonoscopy reports. In the clinical trial, 155 patients had clinically significant polyps. We examined the patients with polyps and identified 28 flat lesions in 25 patients. Flat lesions were determined under either "height" ( $<3 \mathrm{~mm}$ high) or "ratio" (height $<1 / 2$ long axis) criteria. Among them, 11 (38\%) were false negatives in CTC [16]. Lesion sizes ranged from 6-18 $\mathrm{mm}$ with an average of $9 \mathrm{~mm}$ based on optical colonoscopy measurements. 


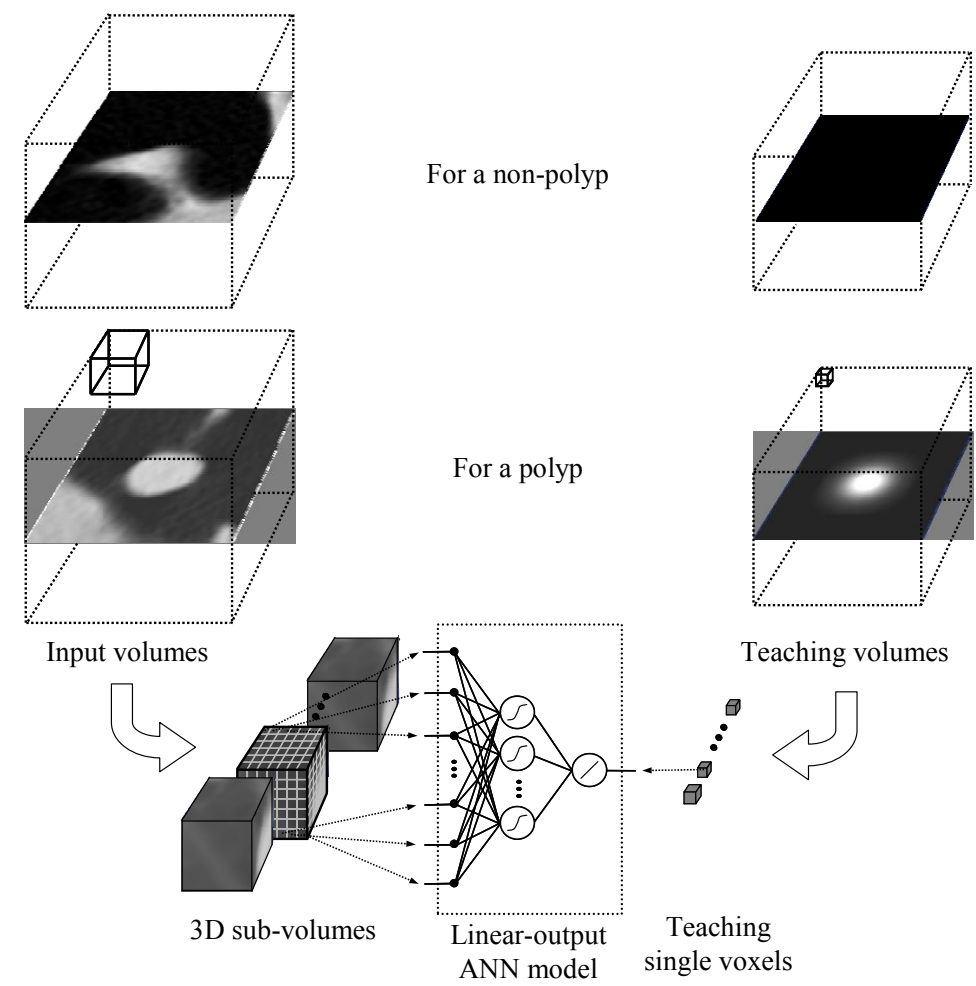

Fig. 1. Architecture and training of a single 3D MTANN consisting of a linear-output multilayer ANN model (i.e., the activation function of the output unit is a linear function instead of a sigmoid function) and a massive-sub-volume training scheme. The input CTC volume including a polyp (which could be a flat lesion) or a non-polyp is divided voxel by voxel into a large number of overlapping 3D sub-volumes. All voxel values in each of the sub-volumes are entered as input to the 3D MTANN, whereas a voxel value at each voxel from the teaching volume is used as the teaching value.

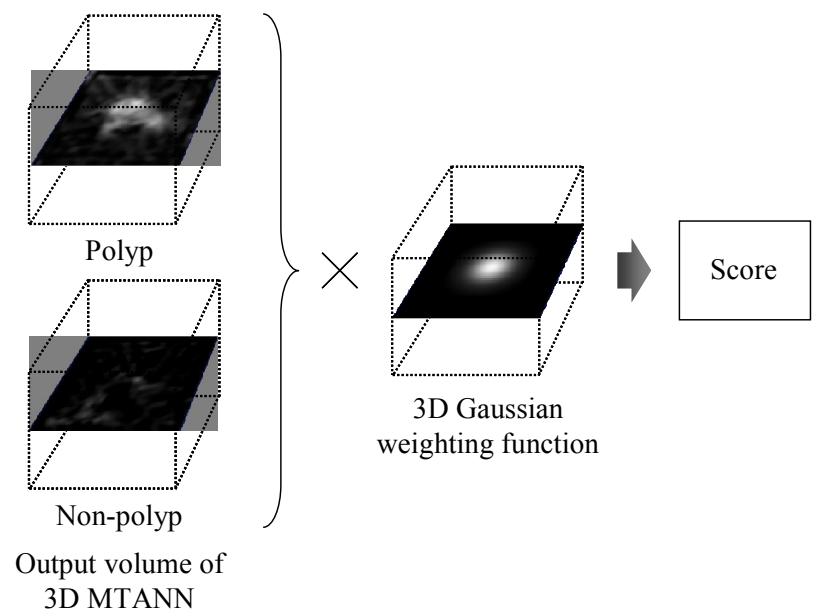

Fig. 2. Schematic illustration of a scoring method for combining output voxels from a trained expert 3D MTANN to obtain a case-based score. 


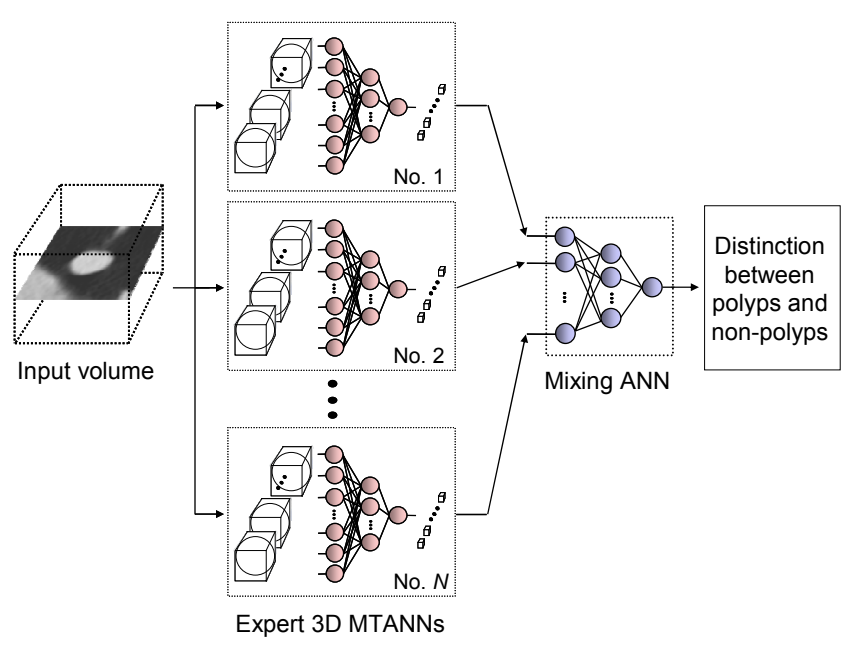

Fig. 3. A mixture of expert 3D MTANNs for distinguishing polyps including flat lesions from various types of FPs. The outputs of the expert 3D MTANNs are combined with a mixing ANN so that the mixture of expert 3D MTANNs can remove various types of non-polyps.

\section{RESULTS}

Our initial polyp-detection scheme without LDA yielded 71\% (20/28) by-polyp sensitivity with 25 (512/25) FPs per patient for the 28 flat lesions including 11 lesions "missed" by reporting radiologists in the original clinical trial. With LDA, 105 FPs were removed with loss of one true positive (TP), thus yielding 68\% (19/28) by-polyp sensitivity with $16.3(407 / 25)$ FPs per patient. We applied the trained expert 3D MTANNs for further reduction of the FPs. The 3D MTANNs were able to remove $39 \%$ (158/407) of the FPs without removal of any TPs. Thus, our CAD scheme achieved a by-polyp sensitivity of 68\% (19/25) with $10(249 / 25)$ FPs per patient, including six of 11 flat lesions "missed" by reporting radiologists in the original trial. Our MTANN CAD scheme detected 67\% (12/18) and 70\% (7/10) of flat lesions ranging from 6-9 $\mathrm{mm}$ and those $10 \mathrm{~mm}$ or larger, respectively, including six lesions "missed" by reporting radiologists in the original trial, with $10(249 / 25)$ FPs per patient. Figure 4 shows examples of flat lesions, which are very small or on a fold (these are major causes of human misses). Some flat lesions are known to be histologically aggressive; therefore, detection of such lesions is critical clinically, but they are difficult to detect because of their uncommon morphology. Our CAD scheme detected these "difficult" flat lesions correctly. It should be noted that these two cases were "missed" by reporting radiologists in the original trial; thus, detection of these lesions may be considered "very difficult." 


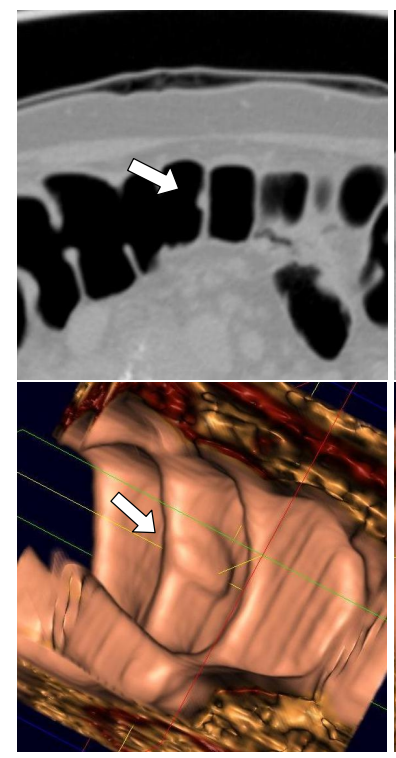

(a)

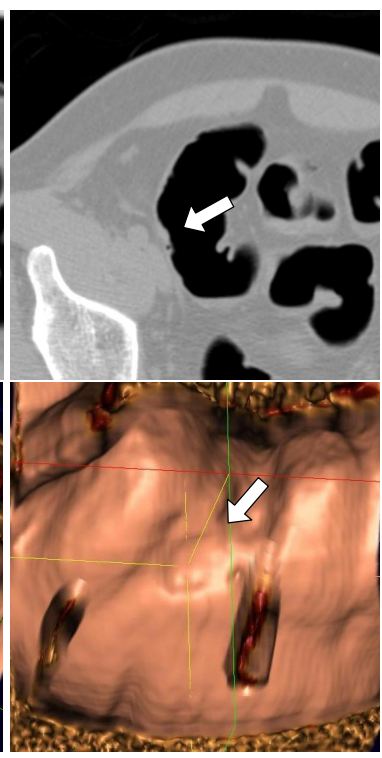

(b)

Fig. 4. Illustrations of flat lesions which exhibit uncommon flat morphology. (a) A flat lesion on a fold (10 mm; adenoma) in the cecum was detected correctly by our CAD scheme (indicated by an arrow). (b) A small flat lesion (6 mm; adenoma) in the cecum was detected correctly by our CAD scheme.

\section{DISCUSSION}

To detect flat lesions, we developed a precise shape analysis to distinguish 3D shapes of objects precisely. This precise shape analysis contributed to a high detection sensitivity of flat lesions. Because of similarity between flat lesions and haustral folds, a larger number of FPs was produced. To distinguish flat lesions from various types of FPs including haustral folds, we developed a mixture of expert 3D MTANNs. The results demonstrated that the mixture of expert 3D MTANNs removed FPs including haustral folds substantially while maintaining the sensitivity of flat lesions. In addition, our flat-lesion database included lesions "missed" by reporting radiologists in the original trial. This study addresses the issue of a CAD scheme applied to flat lesions which existing CAD schemes as well as radiologists are likely to fail to detect.

\section{CONCLUSION}

With our CAD scheme utilizing 3D MTANNs, 68\% (19/28) of flat lesions, including six lesions "missed" by reporting radiologists in the original trial, were detected correctly, with a reasonable number of FPs. Our scheme would be useful for detecting flat lesions which are a major source of false negatives, thus potentially improving radiologists' sensitivity in their detection of polyps in CTC.

\section{ACKNOWLEDGMENT}

The authors are grateful to Ms. E. F. Lanzl for improving the manuscript. This work was supported by an American Cancer Society Illinois Division Research Grant, Grant Number R01CA120549 from the National Cancer Institute/National Institutes of Health, and SIRAF Cluster Funding in research.

\section{REFERENCES}

[1] Fidler, J. and Johnson, C., "Flat polyps of the colon: accuracy of detection by CT colonography and histologic significance," Abdom Imaging (2009).

[2] Ross, A. S. and Waxman, I., "Flat and depressed neoplasms of the colon in Western populations," Am J Gastroenterol 101 (1), 172-80 (2006). 
[3] Soetikno, R. M., Kaltenbach, T., Rouse, R. V., et al., "Prevalence of nonpolypoid (flat and depressed) colorectal neoplasms in asymptomatic and symptomatic adults," JAMA 299 (9), 1027-35 (2008).

[4] Dorai, C and Jain, AK, "COSMOS - A representation scheme for 3D free-form objects," IEEE Trans. Pattern Anal. Mach. Intell. 19 (10), 1115-1130 (1997).

[5] Suzuki, K., Yoshida, H., Nappi, J., et al., "Massive-training artificial neural network (MTANN) for reduction of false positives in computer-aided detection of polyps: Suppression of rectal tubes," Med. Phys. 33 (10), 3814-3824 (2006).

[6] Suzuki, K., Armato, S. G., Li, F., et al., "Massive training artificial neural network (MTANN) for reduction of false positives in computerized detection of lung nodules in low-dose CT," Med. Phys. 30 (7), 1602-1617 (2003).

[7] Suzuki, K., Shiraishi, J., Abe, H., et al., "False-positive reduction in computer-aided diagnostic scheme for detecting nodules in chest radiographs by means of massive training artificial neural network," Acad. Radiol. 12 (2), 191-201 (2005).

[8] Suzuki, K., Li, F., Sone, S., et al., "Computer-aided diagnostic scheme for distinction between benign and malignant nodules in thoracic low-dose CT by use of massive training artificial neural network," IEEE Trans. Med. Imaging 24 (9), 1138-1150 (2005).

[9] Suzuki, K., and Doi, K., "How can a massive training artificial neural network (MTANN) be trained with a small number of cases in the distinction between nodules and vessels in thoracic CT?" Acad. Radiol. 12 (10), 1333-1341 (2005).

[10] Suzuki, K., Abe, H., MacMahon, H., et al., "Image-processing technique for suppressing ribs in chest radiographs by means of massive training artificial neural network (MTANN)," IEEE Trans. Med. Imaging 25 (4), 406-416 (2006).

[11] Suzuki, K., Horiba, I., and Sugie, N., "Neural edge enhancer for supervised edge enhancement from noisy images," IEEE Trans. Pattern Anal. Mach. Intell. 25 (12), 1582-1596 (2003).

[12] Suzuki, K., Horiba, I., and Sugie, N., "Efficient approximation of neural filters for removing quantum noise from images," IEEE Trans. Signal Process. 50 (7), 1787-1799 (2002).

[13] Suzuki, K., Horiba, I., Sugie, N., et al., "Extraction of left ventricular contours from left ventriculograms by means of a neural edge detector," IEEE Trans. Med. Imaging 23 (3), 330-339 (2004).

[14] Suzuki, K., Yoshida, H., Nappi, J., et al., "Mixture of expert 3D massive-training ANNs for reduction of multiple types of false positives in CAD for detection of polyps in CT colonography," Med. Phys. 35 (2), 694-703 (2008).

[15] Rockey, D. C., Paulson, E., Niedzwiecki, D., et al., "Analysis of air contrast barium enema, computed tomographic colonography, and colonoscopy: prospective comparison," Lancet 365 (9456), 305-11 (2005).

[16] Doshi, T., Rusinak, D., Halvorsen, R. A. et al., "CT colonography: False-negative interpretations," Radiology 244 (1), 165-73 (2007). 